PAPER • OPEN ACCESS

Polymer-assisted TEM specimen preparation method for oxidationsensitive 2D materials

To cite this article: Janis Köster et al 2021 Nanotechnology 32075704

View the article online for updates and enhancements. 


\title{
Polymer-assisted TEM specimen preparation method for oxidation-sensitive 2D materials
}

\author{
Janis Köster ${ }^{1}{ }^{\oplus}$, Baokun Liang ${ }^{1}{ }^{\oplus}$, Alexander Storm and Ute Kaiser \\ Electron Microscopy Group of Materials Science, Ulm University, Albert-Einstein-Allee 11, D-89081 Ulm, \\ Germany \\ E-mail: ute.kaiser@uni-ulm.de
}

Received 11 August 2020, revised 8 October 2020

Accepted for publication 26 October 2020

Published 24 November 2020

\begin{abstract}
By structural and analytical TEM and scanning electron microscopy experiments we show that atomically-resolved structural characterization of oxidation-sensitive two-dimensional material is strongly hindered when the final step of the preparation process, the transfer to the TEM grid, is performed with a wet etching method involving bases or acids, interacting with the highly reactive sample surface. Here we present an alternative polymer-assisted and mechanicalexfoliation-based sample preparation method and demonstrate it on selected oxidation-sensitive transition metal phosphorus trisulfides and transition metal dichalcogenides. The analysis, obtained from the samples prepared with both of the methods clearly show that oxidation is the origin of discrepancy, the oxidation during the final preparation step is strongly reduced only when the new method is applied, and atomically-resolved structural characterization of the pristine structures is now possible.
\end{abstract}

Supplementary material for this article is available online

Keywords: 2D material, HRTEM, TMPT, TMD, oxidation-sensitive, specimen preparation

(Some figures may appear in colour only in the online journal)

\section{Introduction}

Nowadays, the number of two-dimensional (2D) materials is fast-growing and transition metal phosphorous trisulfides (TMPTs) are one of the new-born stars due to their magnetic and electric properties [1-3], allocating them as promising candidates for future heterostructure devices [4-6], for instance, combined with members of the material class of transition metal dichalcogenides (TMDs) [7]. Aberrationcorrected high-resolution transmission electron microscopy (AC-HRTEM) offers a powerful approach to analyse and modify freestanding thin samples at the atomic scale [8-12]. However, surface contamination and oxidation hinder the analysis of the material in its pristine state. Thus, the fabrication of high-quality TEM samples is of fundamental importance. In previous studies, many different sample

1 These authors contributed equally to this work. preparation methods were applied for 2D materials: standard transfer with polymer [13], direct transfer for the case of graphene [14], polyimide-assisted transfer for the case of h-BN [15], liquid exfoliation [16], and scotch tape method with potassium hydroxide $(\mathrm{KOH})$. In the following, the scotch tape method with potassium hydroxide will be referred to as the ' $\mathrm{KOH}$ method' [17]. The $\mathrm{KOH}$ method is an effective method to transfer the flake of interest with predetermined thickness to a certain position on the TEM grid, and therefore commonly used in the TEM community. As the $\mathrm{KOH}$ method is conducted in an ambient atmosphere and involves a highly reactive chemical, in this preparation process chemical reactions take place and consequently structural decomposition occurs $[18,19]$. Therefore, TEM imaging of the atomic structure of oxidation-sensitive TMPTs and TMDs, when prepared with the $\mathrm{KOH}$ method, is very difficult. To prevent the materials from oxidation during TEM preparation, a stamping method conducted in an oxygen-free 

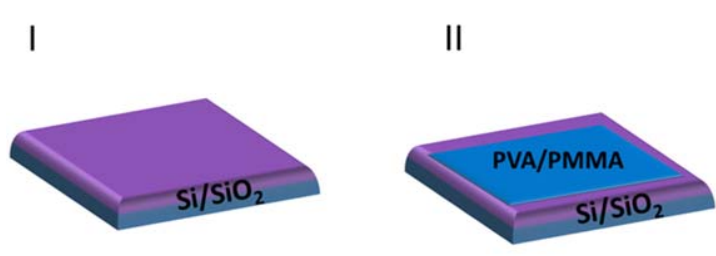

IV

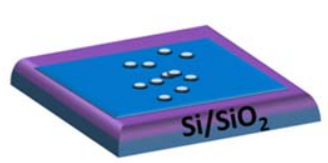

$\mathrm{V}$

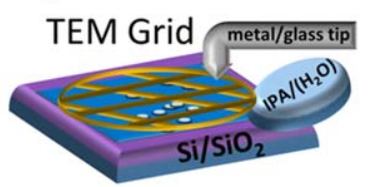

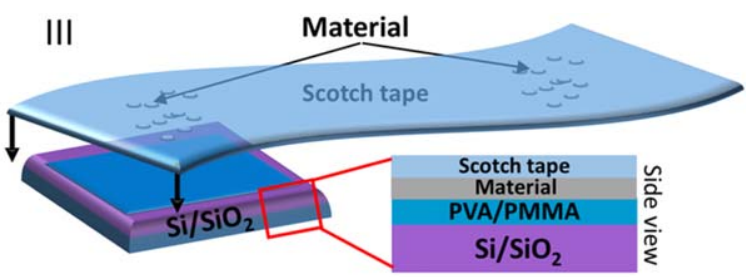

VI

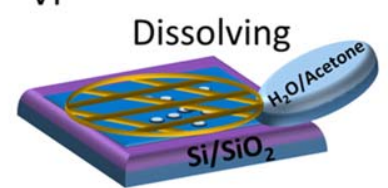

VII

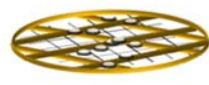

Figure 1. Step by step polymer-assisted preparation of a freestanding TEM sample without base or acid. The substrate is a $\mathrm{Si} / \mathrm{SiO} \mathrm{O}_{2}$ wafer coated with polyvinyl alcohol (PVA) or poly (methyl methacrylate) (PMMA) (shown in I and II). First, the bulk material is thinned down by mechanical exfoliation with scotch tape. By pressing the scotch tape on the wafer (depiction III), the material flakes are deposited on the polymer layer (depiction IV). Subsequently, thin flakes can be identified with an optical microscope. For calculating the contrast of thin layers on a semi-infinite $\mathrm{Si}$ substrate covered with a $\mathrm{SiO}_{2}-\mathrm{PVA} / \mathrm{PMMA}$ layer, a theoretical model based on the Fresnel equation was used [22] (see figure S1). In step V, the TEM grid is brought into contact with the wafer by applying water or IPA, depending on the applied polymer layer. Evaporation and adhesion force of the liquid brings the flexible carbon mesh membrane of the TEM grid in contact with the polymer layer. Further, the grid is removed by dissolving the polymer layer (shown in VI). Type 1 (Ultrapure) water is used in the case of a PVA layer and acetone or anisole in the case of PMMA. In VII the TEM grid with deposited material flakes is illustrated.

environment was developed [20, 21]. This method allows to prepare single-layers of oxidation-sensitive 2D materials. However, it faces the challenge of transferring a freestanding material flake to a TEM grid.

Here we propose a new polymer-assisted method for oxidation-sensitive 2D materials performed under ambient conditions, which minimizes oxidation and allows a controlled transfer to the TEM grid (see figure S1, available online at stacks.iop.org/NANO/32/075704/mmedia). This method is exemplarily performed on $\mathrm{FePS}_{3}, \mathrm{MnPS}_{3}$, $1 \mathrm{~T}-\mathrm{TiSe}_{2}$, and $2 \mathrm{H}-\mathrm{NbSe}_{2}$. In addition, the method maintains the possibility to determine the sample thickness (single-layer or few-layer) before the transfer process.

\section{Proposed sample preparation method}

Figure 1 illustrates the preparation process in detail. Our preparation process starts with thinning down the bulk material by the established mechanical exfoliation technique. The resulting flakes are then deposited on a polymer-coated $\mathrm{Si} / \mathrm{SiO}_{2}$ wafer in which the polymer layer can be either PVA or PMMA. To bring the underlying polymer layer and the sample in contact with the TEM grid, the grid is fixed using a metal or glass tip and wetted from the outside by a drop of isopropyl alcohol (IPA) or water, depending on the polymer layer used. Due to the evaporation of the liquids, the flexible carbon mesh membrane of the TEM grid is directly brought in contact with the material flake. Subsequently, the fixing tool (the metal or glass tip) is removed and the underlying polymer layer is then dissolved by water in the case of PVA, or by acetone if PMMA is used. The most gently way for dissolving the PVA or PMMA layer is to drop the liquids right beside the grid, such that the dissolving process takes place from the edge to the center of the grid. After the underlying polymer layer is dissolved, the grid floats on the liquid drop and can be gently removed by tweezers. This process prevents the material from contacting with acids or bases.

\section{Results and analysis}

In the following, the performance in the sample quality of the polymer-assisted method is analysed and compared to the $\mathrm{KOH}$ method. We start by testing the effect of the $\mathrm{KOH}$ solution on the surface morphology and chemistry of $\mathrm{KOH}-$ treated and untreated bulk $\mathrm{MnPS}_{3}$ flakes using scanning electron microscopy (SEM). In figure 2(a), SEM images of pristine $\mathrm{MnPS}_{3}$ are presented on the left side and after contact with $\mathrm{KOH}$ solution on the right side. Dramatic changes in the surface morphology, for example increased roughness, are visible. In figure 2(b), the corresponding energy-dispersive $\mathrm{x}$-ray (EDX) spectra are shown in the energy range of 0-6.6 $\mathrm{keV}$ taken from the red-framed and blue-framed areas in figure 2(a). The two spectra show similarities as in both spectra carbon $(\mathrm{C}-\mathrm{K} \alpha=0.277 \mathrm{keV})$ and oxygen (O$\mathrm{K} \alpha=0.525 \mathrm{keV}$ ) signals are visible. These peaks may originate from the SEM sample fabrication process, which was performed in air. However, strong differences can be observed in the untreated sample (red curve), the maximum peak ratios of the oxygen peak with respect to the manganese $(\mathrm{Mn}-\mathrm{K} \alpha=5.900 \mathrm{keV}$; Mn-L $\alpha=0.637 \mathrm{keV}$ ), sulfur (S$\mathrm{K} \alpha=2.309 \mathrm{keV})$, and phosphorous $(\mathrm{P}-\mathrm{K} \alpha=2.010 \mathrm{keV})$ peaks of $\mathrm{MnPS}_{3}$ are low, while the ratios in the $\mathrm{KOH}$-treated case (blue curve) are considerably higher. This indicates a stronger oxidation for $\mathrm{MnPS}_{3}$ after contact with the $\mathrm{KOH}$ solution. Additionally, the peak intensities of the $\mathrm{P}$ and $\mathrm{S}$ peaks in the blue curve are lowered with respect to the former ratios between the metal and the elements $\mathrm{P}$ and $\mathrm{S}$ (figure S2 provides cross-section SEM images and the corresponding 


\section{SEM}

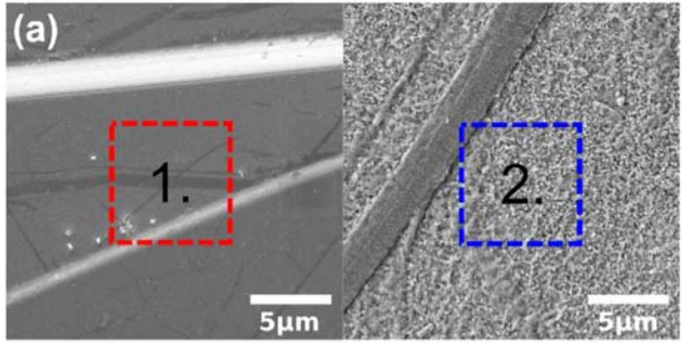

(b)

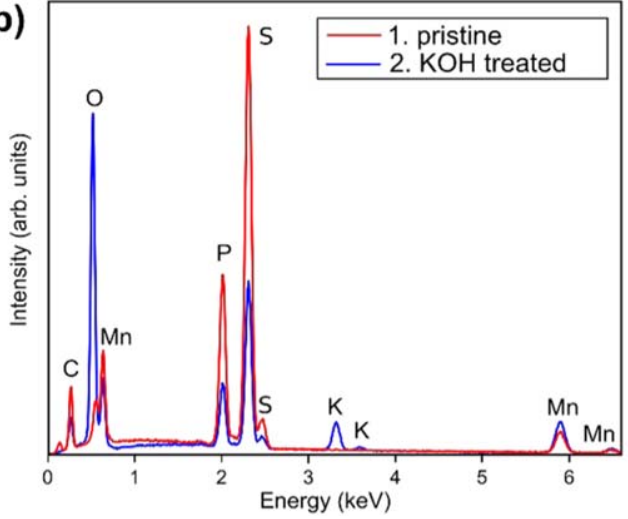

TEM
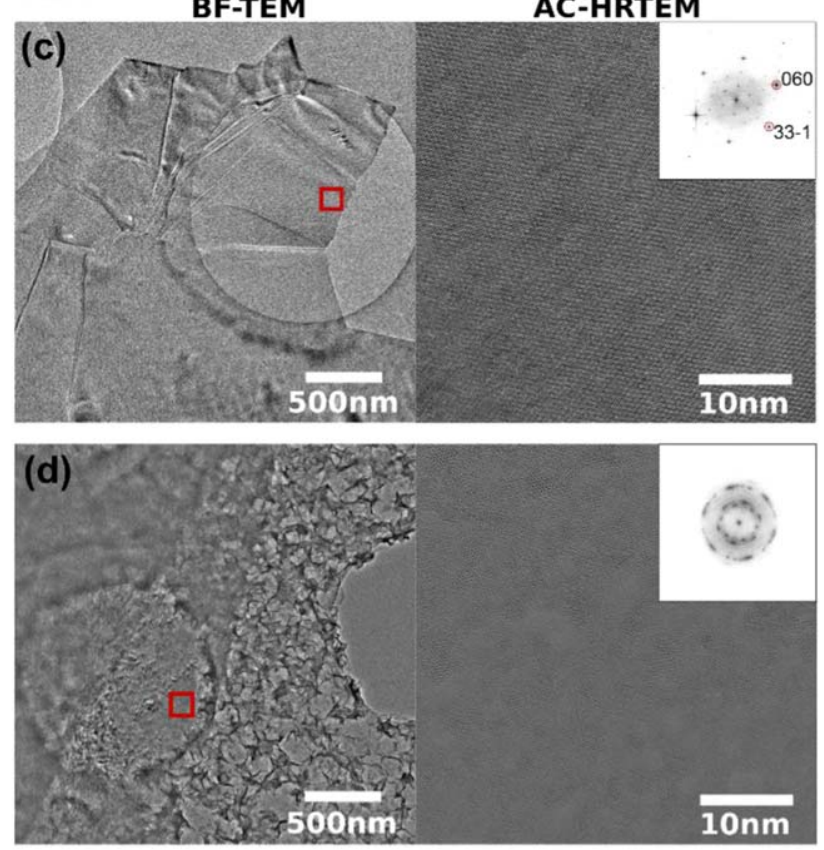

Figure 2. Structural and chemical characterization of bulk (a)-(b) and few-layer $\mathrm{MnPS}_{3}$ (c)-(d). (a) shows SEM images of the surfaces of a pristine (left) and $\mathrm{KOH}$ treated (right) bulk $\mathrm{MnPS}_{3}$ crystal. The marked red and blue squares indicate the corresponding areas for EDX analysis, spectra are shown in (b). The strong $\mathrm{O}$ peak after contact with $\mathrm{KOH}$ is the most obvious difference between both spectra. The peak intensities of the $\mathrm{Mn}(\mathrm{K} \alpha 5.900 \mathrm{keV} / \mathrm{L} \alpha 0.637 \mathrm{keV}), \mathrm{S}(\mathrm{K} \alpha 2.309 \mathrm{keV})$, and $\mathrm{P}(\mathrm{K} \alpha 2.010 \mathrm{keV})$ are clearly visible in both of the spectra. However, the $\mathrm{S}$ and $\mathrm{P}$ peak intensities are strongly reduced after contact with $\mathrm{KOH}$ because of materials decomposition. Moreover, a $\mathrm{K}$ peak is only visible in the $\mathrm{KOH}$ treated sample. (c) BF-TEM overview image along the [103] zone-axis of few-layer MnPS $_{3}$ prepared with the PVA method, the area where the AC-HRTEM image is taken is marked with a red rectangle. The single crystalline nature is clearly visible. (d) BF-TEM overview image of few-layer $\mathrm{MnPS}_{3}$ prepared with the $\mathrm{KOH}$ method, showing heavy damages. From the AC-HRTEM and corresponding FFT images on the right, we see the polycrystalline nature as well as the high degree of amorphisation, right up to the formation of holes in the thin layer (results of $\mathrm{FePS}_{3}$ are presented in figure S5).

EDX spectra of bulk FePS 3 and $\mathrm{MnPS}_{3}$ after the treatment with $\mathrm{KOH}$ solution). Moreover, a potassium (K) peak (K-K $\alpha$ $3.314 \mathrm{keV}$ ) becomes visible for the $\mathrm{KOH}$-treated sample. In the next step, we compared a much thinner $\mathrm{MnPS}_{3}$ sample (few-layer $\mathrm{MnPS}_{3}$ ) prepared with the $\mathrm{KOH}$ method and with the polymer-assisted method using AC-HRTEM (the corresponding structure models are given in figure S3). In the latter case, the overview BF-TEM image of few-layer $\mathrm{MnPS}_{3}$ in figure 2(c) shows an intact flake with sharp edges. The ACHRTEM image, together with corresponding Fast Fourier Transform (FFT) patterns, clearly demonstrates the crystalline nature of the material. On the contrary, the BF-TEM image of the flake prepared with the $\mathrm{KOH}$ method in figure 2(d) shows the deterioration of the flake. The corresponding AC-HRTEM image (taken from the red rectangular in the BF-TEM image) demonstrates the presence of holes and a polycrystalline nature of the material, underlined by the corresponding FFT patterns in the upper right. Results on $\mathrm{FePS}_{3}$ showed amorphisation after the material was treated by the $\mathrm{KOH}$ solution as well (see figure S4 and the more detailed description of the decomposition process).

To prove the adaptability of the proposed polymerassisted preparation method to highly oxidation-sensitive TMDs, we now study $1 \mathrm{~T}-\mathrm{TiSe}_{2}$. Two samples of few-layer $1 \mathrm{~T}-\mathrm{TiSe}_{2}$ are prepared for TEM investigation using the polymers PVA and PMMA as substrate, respectively. The intension of applying two polymers is to find out whether the success of the proposed method is sensitive to the type of the polymer and the different related solvents, water or acetone. The results of BF-TEM, AC-HRTEM, and EELS analysis are presented in figure 3 (additional SEM and EDX results are provided in figure S6). By comparing the overview BF-TEM images, it is clearly seen that the edge of the flake is not sharp and shows a deterioration in the $\mathrm{KOH}$-treated sample, as highlighted by the two red arrows in figure 3(a). The other two samples show sharp edges, see figures 3(b) and (c). Comparing the AC-HRTEM images and their FFTs placed as insets obtained from the red rectangles marked in the corresponding BF-TEM images in figures 3(a)-(c), the differences in the atomic structures are obvious. The crystalline $1 \mathrm{~T}-\mathrm{TiSe}_{2}$ atomic structure is shown in the AC-HRTEM images in figures 3(b) and (c), highlighted by the FFTs (the corresponding structure models are given in figure S3). In contrast, the AC-HRTEM image of the $\mathrm{KOH}$ treated sample in figure 3(a) shows a predominant amorphous structure, as also seen in the FFT at low spatial frequencies. Furthermore, the chemical information of each sample are revealed in the EELS data showing the Ti-L and O-K energy loss edges, see figure 3(d). An oxygen peak starting at $530 \mathrm{eV}$ is only present in the $\mathrm{KOH}$ treated sample [23]. Slight oxidation after PVA 

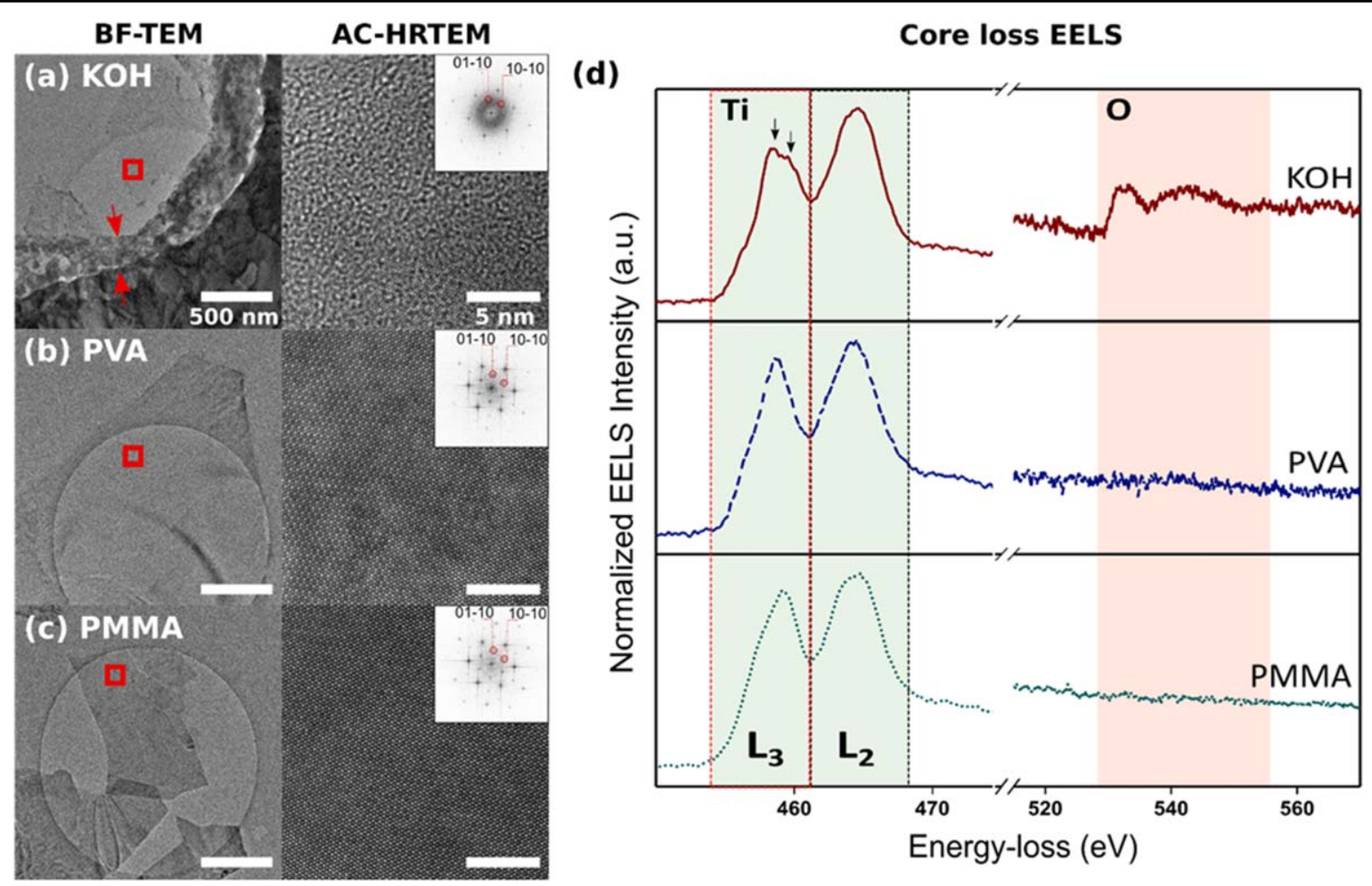

Figure 3. Comparison of the degree of oxidation in few-layer 1T-TiSe 2 prepared by $\mathrm{KOH}$ - and polymer-assisted (PVA and PMMA) methods. Images (a)-(c) show $80 \mathrm{kV}$ overview BF-TEM images on the left (the arrow in (a) marks the edge-contamination) and Cs-corrected HRTEM images on the right along the [001] zone-axis (with their FFTs as insets), obtained from the red-squared areas in the BF-TEM images. By comparing the Cs-corrected HRTEM images in (a)-(c), the oxidation of $\mathrm{TiSe}_{2}$ in (a) can be clearly seen. Accordingly, the corresponding EELS spectra in (d) show that in the case of the $\mathrm{KOH}$ method prepared sample, an $\mathrm{O}$ peak at $530 \mathrm{eV}$ occurs and a splitting of the Ti- $\mathrm{L}_{3}$ edge (see the black arrows) originating from oxidation $\left(\mathrm{TiO}_{2}\right.$ formation) is visible. In the PVA and PMMA method cases, the positions and shapes of the Ti-L2,3 peaks at 462 and $456 \mathrm{eV}$ show just the edge structure for Ti in 1T-TiSe 2 (the raw data of the spectra is given in figure S7).

and PMMA treatment might be expected as well, as they are prepared under ambient conditions. As no O-K peak was measured in EELS, the amount of oxidation must at least be below the signal to noise ratio for $\mathrm{O}$ detection for the settings used to acquire the spectra.

Moreover, the changes in the chemical bonding due to the oxidation are also visible in the $\mathrm{Ti}^{-} \mathrm{L}_{3}$ edges. The $\mathrm{Ti}$ spectra contain bands attributed to the $3 \mathrm{~d} \leftarrow 2 \mathrm{p}_{3 / 2}$ and $3 \mathrm{~d} \leftarrow 2 \mathrm{p}_{1 / 2}$ transitions showing up as two main peaks at around $455-463 \mathrm{eV}$ ( $\mathrm{L}_{3}$ peak) and $463 \mathrm{eV}-470 \mathrm{eV}\left(\mathrm{L}_{2}\right.$ peak), respectively. The fine structure of the Ti- $\mathrm{L}_{2,3}$ edge is determined by the chemical composition and structure of the material. Crystal field splitting leads in the case of pristine $\mathrm{TiSe}_{2}$ to a splitting of the $3 \mathrm{~d}$ orbitals to the lower energy $t_{2 \mathrm{~g}}$ and higher energy $e_{\mathrm{g}}$ levels $[24,25]$. Red and green dotted boxes in figure 3(d) highlight the $\mathrm{L}_{3}$ and $\mathrm{L}_{2}$ edges for the different $\mathrm{TiSe}_{2}$ samples, respectively. The corresponding peaks expected for the transition into the empty $t_{2 g}$ and $e_{g}$ levels merge due to the limited EELS energy resolution of 0.6 $\mathrm{eV}$ and acquisition at room temperature [24,26]. As a result, the weaker $\mathrm{t}_{2 \mathrm{~g}}$ transition appears as a broad shoulder. In figure 3(d), in the case of the $\mathrm{KOH}$ prepared sample, the Ti- $\mathrm{L}_{3}$ peak splits (see black arrows) and an O peak appears thus Ti$\mathrm{O}$ bonds formed. The process of oxidization can be described by the formation of $\mathrm{TiO}_{x} \mathrm{Se}_{2-x}$ and a gradual replacement of Se by $\mathrm{O}$ atoms in the crystal [27]. The split in the $\mathrm{L}_{3}$ peak at the higher energy site might be described as a superposition of the spectra of the oxide with the pristine 1T-TiSe layer. Crystalline $\mathrm{TiO}_{2}$ (e.g. anatase and rutile) [27-29] shows a similar core loss $\mathrm{Ti}\left(\mathrm{L}_{2,3}\right)$ edge fine structure as $1 \mathrm{~T}-\mathrm{TiSe}_{2}$ but shifted by several $\mathrm{eV}$ to higher energies [25]. When the outer layers oxidize and the inner layers of $\mathrm{TiSe}_{2}$ stay intact, a superposition of the titanium oxides $\mathrm{L}_{3}$-edge with the 1T-TiSe $\mathrm{L}_{3}$-edge would result in the observed spectra [27], supplementary information (SI) figures S3 and S5. However, due to the limited energy resolution, unambiguous identification of the titanium oxide structure is not possible. Overall, the shown $\mathrm{L}_{2,3}$ edges for the PVA and PMMA samples coincide with previous reported Ti- $\mathrm{L}_{2,3}$ edges in pristine $1 \mathrm{~T}-\mathrm{TiSe}_{2}[24,26,30]$. Moreover, it should be noticed that there is no significant difference between samples prepared via PVA or PMMA.

We verified the applicability of the polymer-assisted sample preparation method to single-layers using another oxidation-sensitive TMD. $2 \mathrm{H}-\mathrm{NbSe}_{2}$ was chosen as it exhibits superconducting properties and thus excellent TEM sample preparation may be crucial for understanding the property's structural origin [31]. The structural model of $2 \mathrm{H}-\mathrm{NbSe}_{2}$ is shown in figure 4(a). AC-HRTEM experiments were performed for single-layer and few-layer $2 \mathrm{H}-\mathrm{NbSe}_{2}$ using the $\mathrm{Cc} / \mathrm{Cs}$ (chromatic and spherical aberration)-corrected SALVE instrument operated at $80 \mathrm{kV}$ (SEM image and EDX 


\section{(a) $2 \mathrm{H}-\mathrm{NbSe}_{2}$}

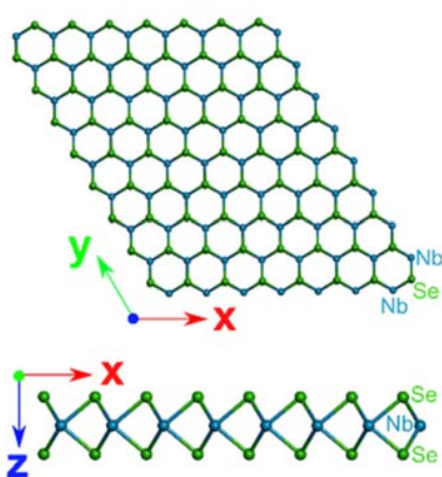

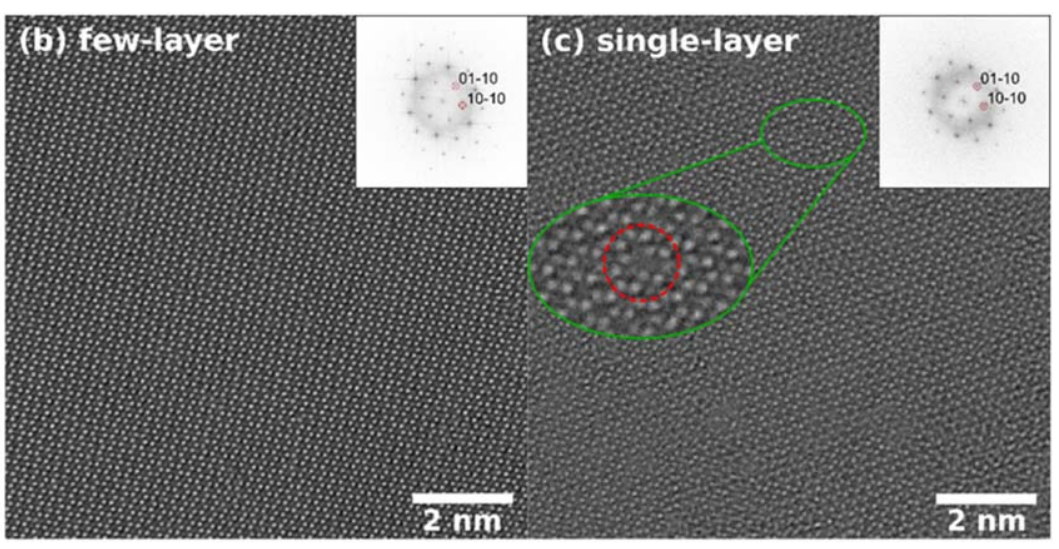

Figure 4. Results of a PVA prepared few-layer and single-layer $2 \mathrm{H}-\mathrm{NbSe}_{2}$ samples. In (a) the structural model of single-layer $2 \mathrm{H}-\mathrm{NbSe} \mathrm{e}_{2}$ is shown in top and side view in which selenium atoms are green and niobium atoms are blue colored. (b) and (c) show $80 \mathrm{kV} \mathrm{Cc/Cs-corrected}$ HRTEM images along the [001] zone-axis demonstrating successful sample preparation without deterioration due to oxidation. FFTs are inserted in the top right corner. A depiction of a selected enlarged region is highlighted by green circles in (c), showing a double Se vacancy (marked by a red dotted circle).

results see figure S6). As figures 4(b) and (c) show, atomically-resolved crystalline structures are visible both for fewlayer and single-layer $2 \mathrm{H}-\mathrm{NbSe}_{2}$, respectively (see figure $\mathrm{S} 1$ for optical images of the single-layer). Atomic defects are also present, which may originate from oxidation during sample preparation and subsequent interaction between the electron beam and the specimen during the imaging process. One defect is enlarged in figure 4(c), representing a double Se vacancy marked by a red dotted circle in the enlarged depiction [31]. Thus it is shown that there is no underlying second layer.

Recent studies showed the sensitivity of $2 \mathrm{H}-\mathrm{NbSe}_{2}$ nanosheets to oxygen [32-34], significantly altering the electronic properties [32]. Therefore, atomically-resolved (S) TEM studies of single-layer $2 \mathrm{H}-\mathrm{NbSe}_{2}$ were only conducted in a graphene-encapsulated 3-layer geometry, as the freestanding single-layer oxidized too fast [33, 34]. Here, atomically-resolved AC-HRTEM characterization of freestanding single-layer $2 \mathrm{H}-\mathrm{NbSe}_{2}$ is reported for the first time.

\section{Summary}

We presented a new, polymer-assisted TEM sample preparation method, which was based on the conventional scotchtape method. Due to the use of polymers, any contact with bases or acids was avoided during transfer of the $2 \mathrm{D}$ sample to the TEM grid. The performance of the proposed method was examined on different oxidation-sensitive TMPTs $\left(\mathrm{MnPS}_{3}\right.$ and $\mathrm{FePS}_{3}$ ) and TMDs (1T-TiSe 2 and $2 \mathrm{H}-\mathrm{NbSe}_{2}$ ). By applying SEM, AC-HRTEM and EELS analysis, a significant increase of the TEM sample quality compared to the $\mathrm{KOH}$ method was demonstrated, which we attributed to strongly reduced oxidation.

We demonstrated that atomically-resolved HRTEM images of the almost pristine crystalline structures can be obtained even of very thin samples such as few-layer $1 \mathrm{~T}-\mathrm{TiSe}_{2}$ and few-layer $2 \mathrm{H}-\mathrm{NbSe}_{2}$, as well as single-layer
$2 \mathrm{H}-\mathrm{NbSe}_{2}$. Moreover, on the example of $1 \mathrm{~T}-\mathrm{TiSe}_{2}$, we showed that the effect of the actual used polymer (PVA and PMMA) and associated solvent (water or acetone) does not influence the TEM sample quality.

In conclusion, our new polymer-assisted TEM sample preparation method provides an enhanced sample quality, enabling atomically precise analysis of structural and chemical properties of oxidation-sensitive freestanding $2 \mathrm{D}$ layers.

\section{Method}

\subsection{Materials and Instrumentations}

In the preparation experiments, the concentration of the $\mathrm{KOH}$ solution was $c(\mathrm{KOH})=0.317 \mathrm{~mol} \mathrm{l}^{-1}$. The PMMA and PVA solutions were produced by dissolving the powder in Type 1 (Ultrapure) anisole and water, respectively. The used concentration was 3\% weight ratio, and the liquids were baked for $24 \mathrm{~h}$ on a hot plate at $60^{\circ} \mathrm{C}-70^{\circ} \mathrm{C}$ with a magnetic stirrer at medium speed in the solution. The thickness of the $\mathrm{SiO}_{2}$ layer on the $\mathrm{Si} / \mathrm{SiO}_{2}$ wafer was $90 \mathrm{~nm} \pm 5 \%$. The SEM measurements were performed with an SEM/FIB Zeiss NVision 40. TEM experiments were conducted with a Cscorrected TEM FEI Titan 80-300 and the Cc/Cs-corrected SALVE (Sub-Ångström Low-Voltage Electron microscopy) instrument operated at $80 \mathrm{kV}$ [35]. For acquiring the spectra, a $50 \mu \mathrm{m}$ selected area aperture was used and the energy resolution during the experiment was determined to be $0.6 \mathrm{eV}$. Furthermore, all experiments were conducted at room temperature. In the SI, an alternative way of conducting our polymer method is illustrated and explained in figure S8.

\section{Acknowledgments}

We would like to thank Manuel Mundszinger for SEM measurements, including the EDX data. We also are grateful to the Institute Laue-Langevin, CS 20156, 38042 Grenoble 
Cedex 9 in France, for the syntheses of the TMPT materials used in this work. We acknowledge funding from the European Union's Horizon 2020 research and innovation programs under Grant Agreement No. 881603 (GrapheneCore3).

\section{ORCID iDs}

Janis Köster (iD https://orcid.org/0000-0001-5123-0170 Baokun Liang (i) https://orcid.org/0000-0002-3725-3285

\section{References}

[1] Léaustic A, Rivière E, Clément R, Manova E and Mitov I 1999 Investigation of the influence of intercalation on the magnetic properties of $\mathrm{Fe}_{x} \mathrm{Cd}_{1-x} \mathrm{PS}_{3}$ layered compounds J. Phys. Chem. B 103 4833-8

[2] Lee J-U, Lee S, Ryoo J H, Kang S, Kim T Y, Kim P, Park C-H, Park J-G and Cheong H 2016 Ising-type magnetic ordering in atomically thin $\mathrm{FePS}_{3}$ Nano Lett. 16 7433-8

[3] Lee S, Choi K Y, Lee S, Park B H and Park J G 2016 Tunneling transport of mono- and few-layers magnetic van der Waals $\mathrm{MnPS}_{3}$ APL Mater. 4 4-11

[4] Gao Y et al 2018 Bias-switchable negative and positive photoconductivity in $2 \mathrm{D} \mathrm{FePS}$ ultraviolet photodetectors Nanotechnology 29244001

[5] Kumar R, Jenjeti R N and Sampath S 2020 Two-dimensional, few-layer MnPS3 for selective $\mathrm{NO}_{2}$ gas sensing under ambient conditions ACS Sens. 5 404-11

[6] Gibertini M, Koperski M, Morpurgo A F and Novoselov K S 2019 Magnetic 2D materials and heterostructures Nat. Nanotechnol. 14 408-19

[7] Huang H, Song J, Yu D, Hao Y, Wang Y and Peng S 2020 Few-layer $\mathrm{FePS}_{3}$ decorated with thin $\mathrm{MoS}_{2}$ nanosheets for efficient hydrogen evolution reaction in alkaline and acidic media Appl. Surf. Sci. 525146623

[8] Gupta A, Sakthivel T and Seal S 2015 Recent development in 2D materials beyond graphene Prog. Mater. Sci. 73 44-126

[9] Zhao X, Kotakoski J, Meyer J C, Sutter E, Sutter P, Krasheninnikov A V, Kaiser U and Zhou W 2017 Engineering and modifying two-dimensional materials by electron beams MRS Bull. 42 667-76

[10] Zhang D, Zhu Y, Liu † L, Ying X, Hsiung C-E, Sougrat R, Li K and Han Y 2018 Atomic-resolution transmission electron microscopy of electron beam-sensitive crystalline materials Science 359 675-9

[11] Lehnert T, Ghorbani-Asl M, Köster J, Lee Z, Krasheninnikov A V and Kaiser U 2019 Electron-beamdriven structure evolution of single-layer $\mathrm{MoTe}_{2}$ for quantum devices ACS Appl. Nano Mater. 2 3262-70

[12] Wang X et al 2014 Chemical vapor deposition growth of crystalline monolayer $\mathrm{MoSe}_{2}$ ACS Nano 8 5125-31

[13] Lin J, Lin Y, Wang X, Xie L and Suenaga K 2017 Gentle transfer method for water- and acid/alkali-sensitive 2D materials for (S) TEM study APL Materials 4116108

[14] Regan W et al 2010 A direct transfer of layer-area graphene Appl.Phys.Lett. 96113102

[15] Pacilé D, Meyer J C, Girit Ç Ö, Zettl A, Pacilé D, Meyer J C, Girit Ç Ö and Zettl A 2008 The two-dimensional phase of boron nitride: few-atomic-layer sheets and suspended membranes Appl. Phys. Lett. 92133107

[16] Nicolosi V, Chhowalla M, Kanatzidis M G, Strano M S and Coleman J N 2013 Liquid exfoliation of layered materials Science 3401226419
[17] Meyer J C, Girit C O, Crommie M F and Zettl A 2008 Hydrocarbon lithography on graphene membranes Appl. Phys. Lett. 92 8-11

[18] Her M, Beams R and Novotny L 2013 Graphene transfer with reduced residue Phys. Lett. A 377 1455-8

[19] Yeh C H, Teng P Y, Chiu Y C, Hsiao W T, Hsu S S H and Chiu P W 2019 Gigahertz field-effect transistors with CMOS-compatible transfer-free graphene ACS Appl. Mater. Interfaces 11 6336-43

[20] Zomer P J, Dash S P, Tombros N and Van Wees B J 2011 A transfer technique for high mobility graphene devices on commercially available hexagonal boron nitride Appl. Phys. Lett. 99 2009-12

[21] Frisenda R, Navarro-Moratalla E, Gant P, Pérez De Lara D, Jarillo-Herrero P, Gorbachev R V and Castellanos-Gomez A 2018 Recent progress in the assembly of nanodevices and van der Waals heterostructures by deterministic placement of 2D materials Chem. Soc. Rev. 47 53-68

[22] Blake P, Hill E W, Neto A H C, Novoselov K S, Jiang D, Yang R, Booth T J, Geim A K, Blake P and Hill E W 2007 Making graphene visible Appl. Phys. Lett. 91063124

[23] Bastow T J, Botton G A, Etheridge J, Smith M E and Whit H J 1999 A study of $\mathrm{Li}_{2} \mathrm{TiOSiO}_{4}$ and $\mathrm{Li}_{2} \mathrm{TiOGeO}_{4}$ by x-ray powder and electron single-crystal diffraction, ${ }^{17} \mathrm{O}$ MAS NMR and $\mathrm{O}$ K-edge and Ti $\mathrm{L}_{2,3}$-edge EELS Acta Cryst. $\mathbf{A 5 5}$ 127-32

[24] Qiao Q, Zhou S, Tao J, Zheng J-C, Wu L, Ciocys S T, Iavarone M, Srolovitz D J, Karapetrov G and Zhu Y 2017 Anisotropic charge density wave in layered $1 \mathrm{~T}-\mathrm{TiSe}_{2}$ Phys. Rev. Mater. 1054002

[25] Fronzoni G, De Francesco R, Stener M and Causà M 2006 Xray absorption spectroscopy of titanium oxide by time dependent density functional calculations J. Phys. Chem. B 110 9899-907

[26] Kobayashi K and Yasuda H 2018 Formation of a superstructure in $1 \mathrm{~T}-\mathrm{TiSe}_{2}$ induced at room temperature by electron beam irradiation Mater. Res. Express 58

[27] Sun L et al 2017 Suppression of the charge density wave state in two-dimensional 1T-TiSe ${ }_{2}$ by atmospheric oxidation Angew. Chem.-Int. Ed. 56 8981-5

[28] de la Peña F, Berger M H, Hochepied J F, Dynys F, Stephan O and Walls M 2011 Mapping titanium and tin oxide phases using EELS: an application of independent component analysis Ultramicroscopy 111 169-76

[29] Kucheyev S O, Van Buuren T, Baumann T F, Satcher J H, Willey T M, Meulenberg R W, Felter T E, Poco J F, Gammon S A and Terminello L J 2004 Electronic structure of titania aerogels from soft x-ray absorption spectroscopy Phys. Rev. B 69 1-7

[30] Monney C et al 2012 Mapping of electron-hole excitations in the charge-density-wave system $1 \mathrm{~T}-\mathrm{TiSe}_{2}$ using resonant inelastic x-ray scattering Phys. Rev. Lett. 109 1-5

[31] Xi X, Zhao L, Wang Z, Berger H, Forró L, Shan J and Mak K F 2015 Strongly enhanced charge-density-wave order in monolayer $\mathrm{NbSe}_{2}$ Nat. Nanotechnol. 10 765-9

[32] Park H, Kim J Y, Oh J Y and Lee T I 2020 Long-term stable $\mathrm{NbSe}_{2}$ nanosheet aqueous ink for printable electronics Appl. Surf. Sci. 504144342

[33] Wang H et al 2017 High-quality monolayer superconductor $\mathrm{NbSe}_{2}$ grown by chemical vapour deposition Nat. Commun. 8 1-8

[34] Nguyen L et al 2017 Atomic defects and doping of monolayer $\mathrm{NbSe}_{2}$ ACS Nano 11 2894-904

[35] The Sub-Angstrom Low-Voltage Electron Microscopy Project www.salve-project.de 